\title{
A THZ Tunable Metamaterial Reflective Polarization Converter Based Vanadium Oxide Film
}

\section{Fuyuan Yu ( $\nabla$ fuyhfut@126.com )}

Huainan Normal University https://orcid.org/0000-0002-7300-9925

\section{Xiongjun Shang}

Changsha University of Science and Technology

\section{Wei Fang}

Huainan Normal University

\section{Qingqing Zhang}

Huainan Normal University

\section{Yan Wu}

Huainan Normal University

\section{Wang Zhao}

Huainan Normal University

Jiabing Zhu

Huainan Normal University

Xiaobo Shen

Huainan Normal University

\section{Research Article}

Keywords: Polarization converter, Vanadium dioxide, Metamaterial

Posted Date: July 30th, 2021

DOI: https://doi.org/10.21203/rs.3.rs-640647/v1

License: (c) (i) This work is licensed under a Creative Commons Attribution 4.0 International License.

Read Full License 


\title{
A THZ tunable metamaterial reflective Polarization Converter Based Vanadium Oxide film
}

Fu-yuan $\mathrm{Yu}^{1}$, Xiong-jun Shang ${ }^{2}$, Wei Fang ${ }^{1}$, Qing-qing Zhang ${ }^{1}$, Yan $\mathrm{Wu}^{1}$, Wang Zhao ${ }^{1}$, Jia-bing Zhu ${ }^{1 *}$, Xiao-bo Shen ${ }^{1 *}$

${ }^{1}$ School of Electronic Engineering, Huainan Normal University, Huainan 232038, People’s Republic of China

${ }^{2}$ Hunan Provincial Key Laboratory of Flexible Electronic Materials Genome Engineering, School of Physics and Electronic Science, Chang sha University of Science and Technology, Changsha 410004, People's Republic of China

*Email: zjb3617@163.com and shenhnnu@163.com

\begin{abstract}
In this paper a simple and tunable reflective polarization converter has been investigated numerically based on metamaterial which composes of a two-corner-cut square patch resonator with a slit embedded into Vanadium dioxide film (VO2) and reflective ground layer. All the results obtained by the CST Microwave Studio show that the polarization conversion ratio (PCR) above $90 \%$ is achieved from $2.22-5.42 \mathrm{THz}$ at the temperature about $25^{\circ} \mathrm{C}$ under the linearly and circularly polarized wave incidence normally. In addition, the influences on electromagnetic polarization properties have been demonstrated with the insulator-to-metal phase transition of the Vanadium dioxide film (VO2) film by the method of varying the temperature. At the same time, to be demonstrated, the physical mechanism of changeable polarization conversion has been discussed by the distributions of current densities. According to the results, the designed metamaterial could be applied in the area of temperature-controlled sensing, $\mathrm{THz}$ wireless communication, tunable polarized devices.
\end{abstract}

Keywords: Polarization converter, Vanadium dioxide, Metamaterial

\section{Introduction}

Polarization is an enormous important property of the electric-magnetic wave for providing real application in information processing, imaging and sensing [1, 2]. As a fundamental phenomenon, polarization converter, that is traditionally accomplished by natural birefringent material or dichroic crystals, has recently acquired much attentions from researchers in different areas. The results can occur attributed to the advent of Metamaterials (MMs). The artificial electromagnetic materials (MMs) not only overcome shortcomings of the effect weakly and the volume bulky incurred by 
traditional materials but also open a promising platform to control the polarization states of light [3, 4]. With regard to MMs converter, many interesting phenomena have been reported in $\mathrm{THz}$ spectrum, such as dual-band [5], tri-band [6], multi-band [7] and broadband [8, 9] polarization converter. However, there are only a few attempts to realize dynamic modulation.

Actually, dynamic response materials including GeSbTe (GST) [10], graphene [11], semiconductors [12], vanadium dioxide (VO2) [13-16], which can be excited by external factors, for instance electric bias, temperature, photo excitation, are capable of accomplishing the modulation. The MMs integrated with dynamic inclusions can accomplish tunable polarization conversion modulation. At the present, the thermal- controlled tunable MMs have been seldom investigated [17, 18]. In respect of temperature-controlled phase change MMs, VO2, as a novel phase-controlled material, exhibits an insulator-to-metal phase transition $[19,20]$ with conductivity of VO2 changes depending on the various temperatures. Hence, in certain perspective, it is possible and worthwhile that tunable polarization conversion can be achieved by the phase transition of VO2.

In this work, a simple and tunable reflective polarization converter based on metamaterial is proposed in $\mathrm{THz}$ spectrum, which composes of a two-corner-cut square patch resonator with a slit embedded into Vanadium dioxide film (VO2) and reflective ground layer. Numerical calculation shows that reflective curve for both linear polarization and circular polarization incidence can hold the same trend with PCR above $90 \%$ from 2.22 to $5.42 \mathrm{THz}$. In addition, to obtain tunable polarization conversion performance, the polarization responses with the respect to the various conductivities of VO2 have been investigated in detail. At last, to be interested, the surface current densities depending on different conductivity from $200,10^{4}$ to $10^{5} \mathrm{~S} / \mathrm{m}$ have also been researched. Therefore, such dynamic changes of phase transition metamaterial offer a new route for acquiring multifunctional devices, such as switches, polarization modulators.

\section{Descriptions of the Designed MMs and Simulation Model}



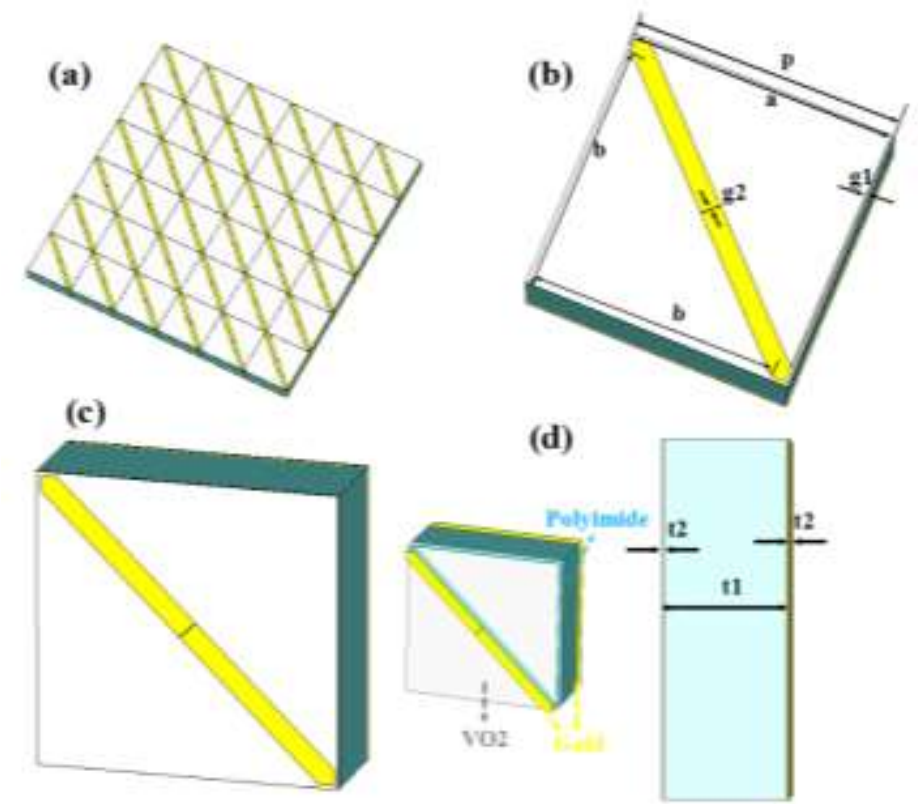

Fig. 1. Schematic diagram of the designed polarization converter. (a) titled extended periodic array view, (b) magnified front view of a unit cell, (c) perspective view of a unit cell, (d) right view of a unit cell.

The proposed MMs (Fig. 1(a)) consist of periodical identical unit cells (Fig. 1(b)) which composed of hybrid layer and metallic ground layer separated by polyimide dielectric layer with a relative dielectric constant of 3.5 [21] and a thickness (t1) of 10um (Fig. 1(d)), as schematically shown in Fig. 1(c). It is obviously that the hybrid layer (first layer) comprises a metallic two-corner-cut square patch resonator with a slit and VO2 film. The top and bottom metallic layers are made by the same gold dealt with the Drude model $\left(\omega_{p}=2 \pi \times 2175 \mathrm{TH} z, \omega_{c}=2 \pi \times 4.35 \mathrm{TH} z\right)[22]$ and a thickness ( $\mathrm{t} 2$ ) of $0.2 \mathrm{um}$. Among the hybrid layer, the properties of $\mathrm{VO} 2$ film can be expressed by the Bruggeman effective model [23]:

$$
\varepsilon(\mathrm{vO} 2)=\frac{1}{4}\left\{\varepsilon_{d}(2-3 \mathrm{~V})+\varepsilon_{m}(3 \mathrm{~V}-1)+\sqrt{\left[\varepsilon_{d}(2-3 \mathrm{~V})+\varepsilon_{m}(3 \mathrm{~V}-1)^{2}+8 \varepsilon_{d} \varepsilon_{m}\right]}\right\},
$$

Where $\varepsilon_{d}$ and $\varepsilon_{m}$ denote dielectric constants of the insulating and metallic phase, respectively. Moreover, $\mathrm{V}$ indicates the volume fraction of the metallic regions. According the above parameters given, the dielectric constant of $\mathrm{VO} 2$ can be obtained arbitrary. In our simulation, the relative permittivity of VO2 can be set as 9 [23], in which the conductivity is $200 \mathrm{~S} / \mathrm{m}$ at the temperature about $25{ }^{\circ} \mathrm{C}$ [23]. All the designs, of which the relevant other parameter given below (Table 1), can be carried out by CST Microwave Studio accomplished by frequency domain solver. Periodic 
boundary condition and port boundary condition are applied to $\mathrm{X} / \mathrm{Y}$ direction and $\mathrm{Z}$ direction, respectively.

Table 1. All dimension parameters of the designed polarization converter

\section{$\begin{array}{llllllll}\text { Parameters } & \text { p } & \text { a } & \text { b } & \text { g1 } & \text { g2 } & \text { t1 } & \text { t2 }\end{array}$}

\section{$\begin{array}{llllllll}\text { Values }(\mu \mathrm{m}) & 30 & 29.2 & 27.6 & 0.4 & 0.05 & 10 & 0.2\end{array}$}

To better understand the performance of the designed polarization converter, the polarization conversion ratio (PCR) can be adopted. Concerning the polarization of incident wave exists along the y-direction, the PCR can be calculated by Eq. (2) as follow. Moreover, for the x-polarized wave incidence, the subscripts about $\mathrm{x}$ and $\mathrm{y}$ in Eq. (2) can be merely interchanged:

$$
P C R=\frac{\left|r_{x y}\right|^{2}}{\left|r_{x y}\right|^{2}+\left|r_{y y}\right|^{2}},
$$

Where $r_{x y}=E_{r x} / E_{i y}$ and $r_{y y}=E_{r y} / E_{i y}$ are reflective coefficients for cross-polarized and co-polarized wave. The alphabet $i$ and $r$ denote incidence and reflection, respectively. For the incident circular polarization, the subscripts about $y$ and $x$ can be replaced $L$ and $R$, respectively.

\section{Results and discussions}

To characterize the performance of the designed MMs, the reflective amplitude $\left(\left|r_{i j}\right|\right)$ and $\mathrm{PCR}_{\mathrm{i}}$ for linear and circular polarization incident wave have been investigated, respectively. The subscript $\mathrm{i}$ and j represent either $\mathrm{x}$, y or R, L. As exhibited in Fig. 2(a), cross-reflective coefficients $\left|r_{x y}\right|$ and $\left|r_{y x}\right|$ indicate the same characteristics which hold above 0.85 from around 2.22 and $5.42 \mathrm{THz}$. While, both the co-polarized reflective coefficients $\left|r_{x x}\right|$ and $\left|r_{y y}\right|$ are much lower than cross-polarized $\left|r_{y x}\right|$ and $\left|r_{x y}\right|$, where beneath 0.07 at the nearby frequency of $2.39,3.78$, ad 5.27THz. At the same time, the curves of cross-reflection coefficients exist three resonant peaks at 2.39, 3.78 an-d 5.27THz. In other words, nearly total the $\mathrm{y}-(\mathrm{x}-)$ polarized wave could be converted the cross polarized, i.e., $\mathrm{x}-(\mathrm{y}-)$ polarized wave. The further results can also be inferred from Fig. 2(b) that the PCR more than 0.99 stands at the nearby frequency points of $2.39,3.78$ and $5.27 \mathrm{THz}$ due to the resonant effects, while the PCR 
more than 0.9 is achieved from vicinity of $2.22-5.42 \mathrm{THz}$. What is more, it is more interesting phenomenon from Fig. 2(c) and (d) that the reflective parameters under the circular polarized wave keep same trends with the reflective parameters under the linear polarized wave, namely $\left|r_{x y}\right|=\left|r_{y x}\right|=\left|r_{R L}\right|=\left|r_{L R}\right|,\left|r_{x x}\right|=\left|r_{y y}\right|=\left|r_{R R}\right|=\left|r_{L L}\right|$ and $\operatorname{PCR}(\mathrm{y})=\mathrm{PCR}(\mathrm{x})=\mathrm{PCR}(\mathrm{R})=\mathrm{PCR}(\mathrm{L})$. Based on analysis above, the designed MMs can be acted as linear polarization as well as circular polarization converter.
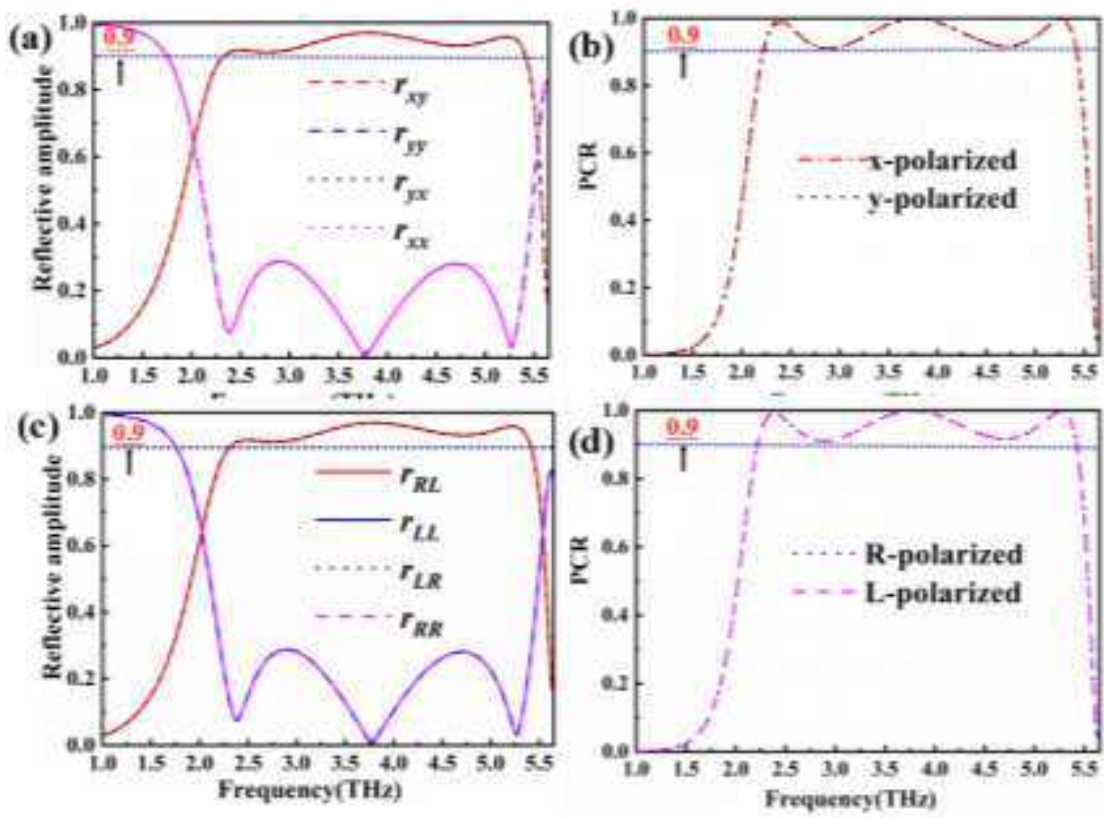

Fig. 2. (a) Simulated reflective coefficient and (b) PCR for incident linear polarization. (c) Simulated reflective coefficient and (d) PCR for incident circular polarization.

To better understand the polarization state of electromagnetic wave under normal illumination, the formula can be expressed as follow [22]:

$$
\begin{aligned}
& \theta=\frac{1}{2} \arctan \left(\frac{2 p_{r} \cos \left(\varphi_{r}\right)}{1-\left|p_{r}\right|^{2}}\right), \\
& \eta=\frac{1}{2} \arcsin \left(\frac{2 p_{r} \sin \left(\varphi_{r}\right)}{1+\left|p_{r}\right|^{2}}\right),
\end{aligned}
$$

Where $p_{r}=\left|r_{x y}\right| /\left|r_{y y}\right|$ and $\varphi_{r}=\arg \left(r_{x y}\right)-\arg \left(r_{y y}\right)$. The $\theta$ denotes the angle between reflective and incident polarization plane, while the $\eta$ characterizes the polarization state of the reflective wave. When the $\eta=0^{\circ}$ and $\theta \neq 90^{\circ}$, the reflective wave keep linear polarization state with an angle of $\theta$ referred to the incident wave, but not cross-polarization state. What is more, the pure cross-polarization wave can be obtained when $\eta=0^{\circ}$ and $\theta=90^{\circ}$. As is shown in Fig. 3, the ellipticity $\eta$ of the incident y-polarized 
wave stands less than $18^{\circ}$ from 2.22 to $5.42 \mathrm{THz}$ at whole frequency band and keeps nearly $0^{\circ}$ at the around resonant frequency of 2.39, 3.78 and 5.27THz. The deep meaning implies that a near linear polarization wave is obtained around the three resonant frequency. Moreover, Furtherly the cross-polarization conversion can be maintained when the polarization azimuth angle $\theta$ keeps $\pm 90^{\circ}$ at the same three resonate frequency points in Fig. 3.

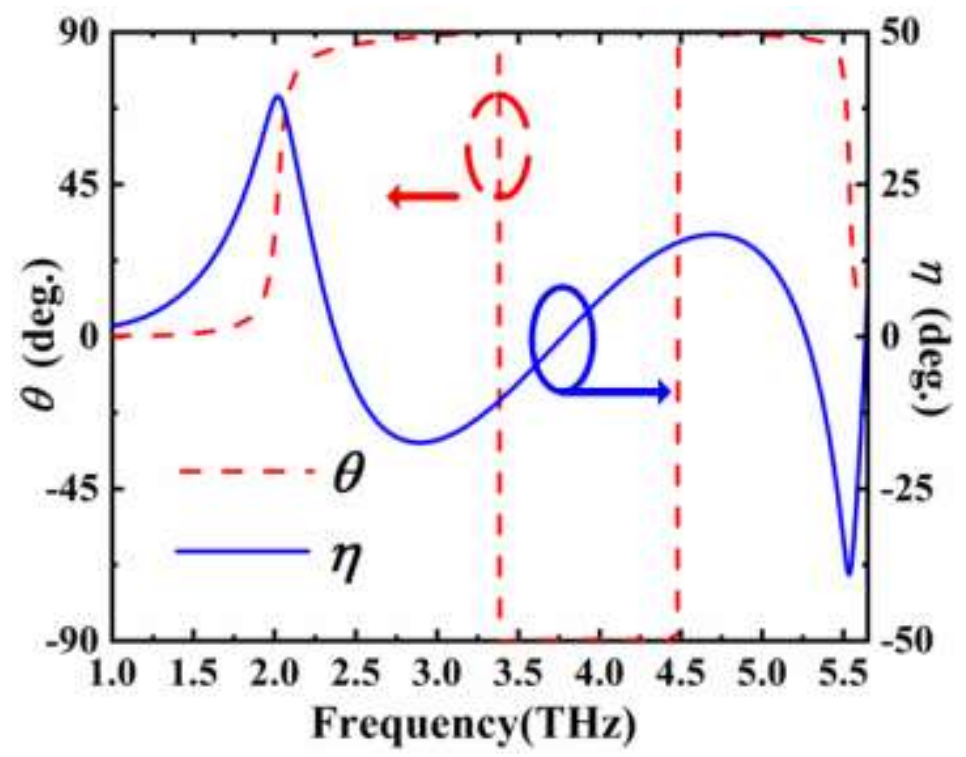

Fig. 3. The polarization azimuth angle $(\theta)$ and ellipticity $(\eta)$ under the normal incident y-polarized wave

To further depict the effects of interesting dynamic tunable reflective polarization conversion for the enhanced hybrid metamaterial, much attentions have been paid on the specific kind thermal material, i.e., VO2. As we all know, the conductivities for VO2 film change with the changeable temperature, thus can result into incident electromagnetic wave phase-transition when VO2 film acts as from insulator state to metallic state. To be concreted, the VO2 film exhibits an insulating state when the value for conductivity is lower than $200 \mathrm{~S} / \mathrm{m}$ controlled by the temperature labelled with $25{ }^{\circ} \mathrm{C}$, while show an metallic state with the conductivity exceed higher than $10^{5} \mathrm{~S} / \mathrm{m}$ attained at the temperature of $85{ }^{\circ} \mathrm{C}$ [23]. To be convinced, as is demonstrated in Fig. 4, the PCR for incident y-polarized wave decreases obviously with the conductivity increasing from 200 to $10^{5} \mathrm{~S} / \mathrm{m}$. Eventually, the PCR is almost 0 when the VO2 film is metal state with the conductivity set as $10^{5}$ $\mathrm{S} / \mathrm{m}$. That is almost all of the incident y-polarized component penetrate through the top hybrid layer and could be transformed into X-polarized component when $\sigma_{\mathrm{VO} 2}=200 \mathrm{~S} / \mathrm{m}$ at the temperature of 
$25{ }^{\circ} \mathrm{C}$. However, the incident y-polarized component reflects by the top hybrid layer when $\sigma_{\mathrm{VO} 2}=10^{5}$ $\mathrm{S} / \mathrm{m}$ at the temperature of $85^{\circ} \mathrm{C}$. According to the analysis above, it can be concluded that the MMs play a role in thermal-controlled switch devices in the area of polarization conversion.

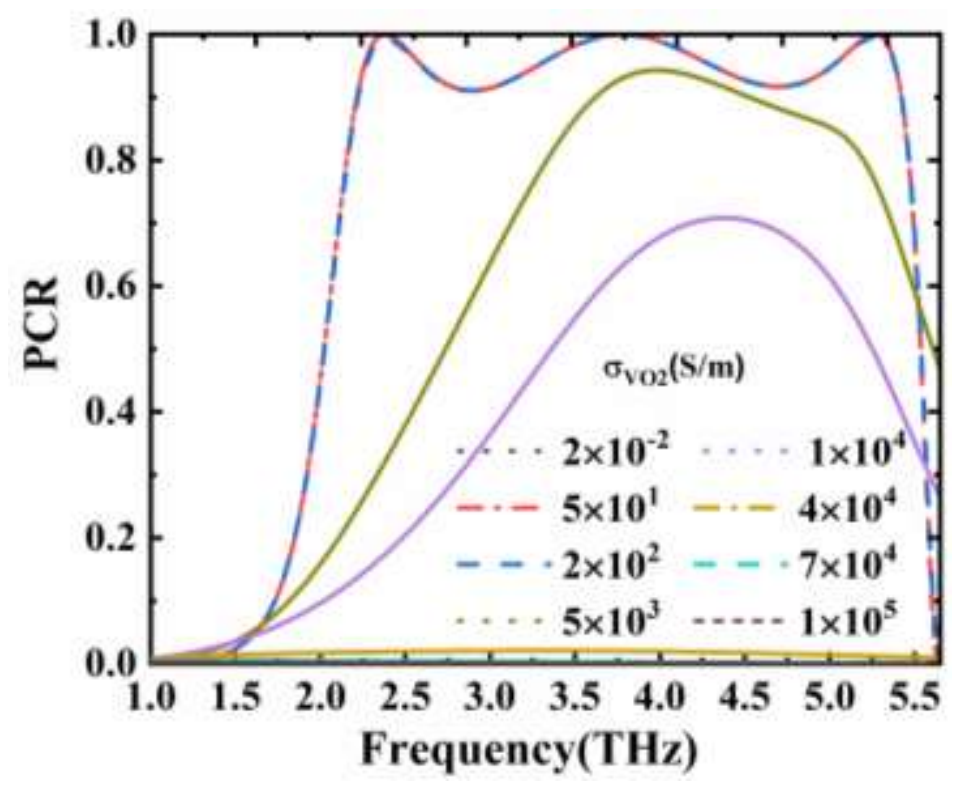

Fig. 4. The PCR of incident y-polarized wave under the different VO2 film conductivities

To understand the physic mechanism, the current densities of incident y-polarized wave are depicted as is shown in Fig. 5. The conductivity of $200,10^{4}$ and $10^{5} \mathrm{~S} / \mathrm{m}$ at $5.27 \mathrm{THz}$ are taken as example to describe thermal control effects. It is obviously that strong parallel currents called electric resonance [24-28] can be observed between the top and bottom layer in Fig. 5(a) and (b) at the conductivity of $200 \mathrm{~S} / \mathrm{m}$. The decomposed Ex component of induced E contributes to cross-polarization conversion. The $\mathrm{E}_{\mathrm{y}}$ component of induced $\mathrm{E}$ has no impact on polarization for its same direction with incident $E_{i}$ [29]. However, the current densities reflect gradually weakly between top and bottom layer when $\sigma_{\mathrm{VO} 2}$ increases from $10^{4}$ to $10^{5} \mathrm{~S} / \mathrm{m}$ as is shown in Fig. 5(c), (d) and (e), (f). Moreover, induced E rotates parallel to incident $E_{\mathrm{i}}$ gradually from Fig. 5(c), (d) to (e), (f). Thus, it can be inferred that the incident wave can change from penetrating state to not penetrating the top layer state with utilizing the thermal controllable VO2. In other words, as the temperature increases, the $\mathrm{y}$ to $\mathrm{x}$ cross-polarized component conversion decrease sharply. The thermal tunable method provides a new route to promote $\mathrm{THz}$ polarization devices. 


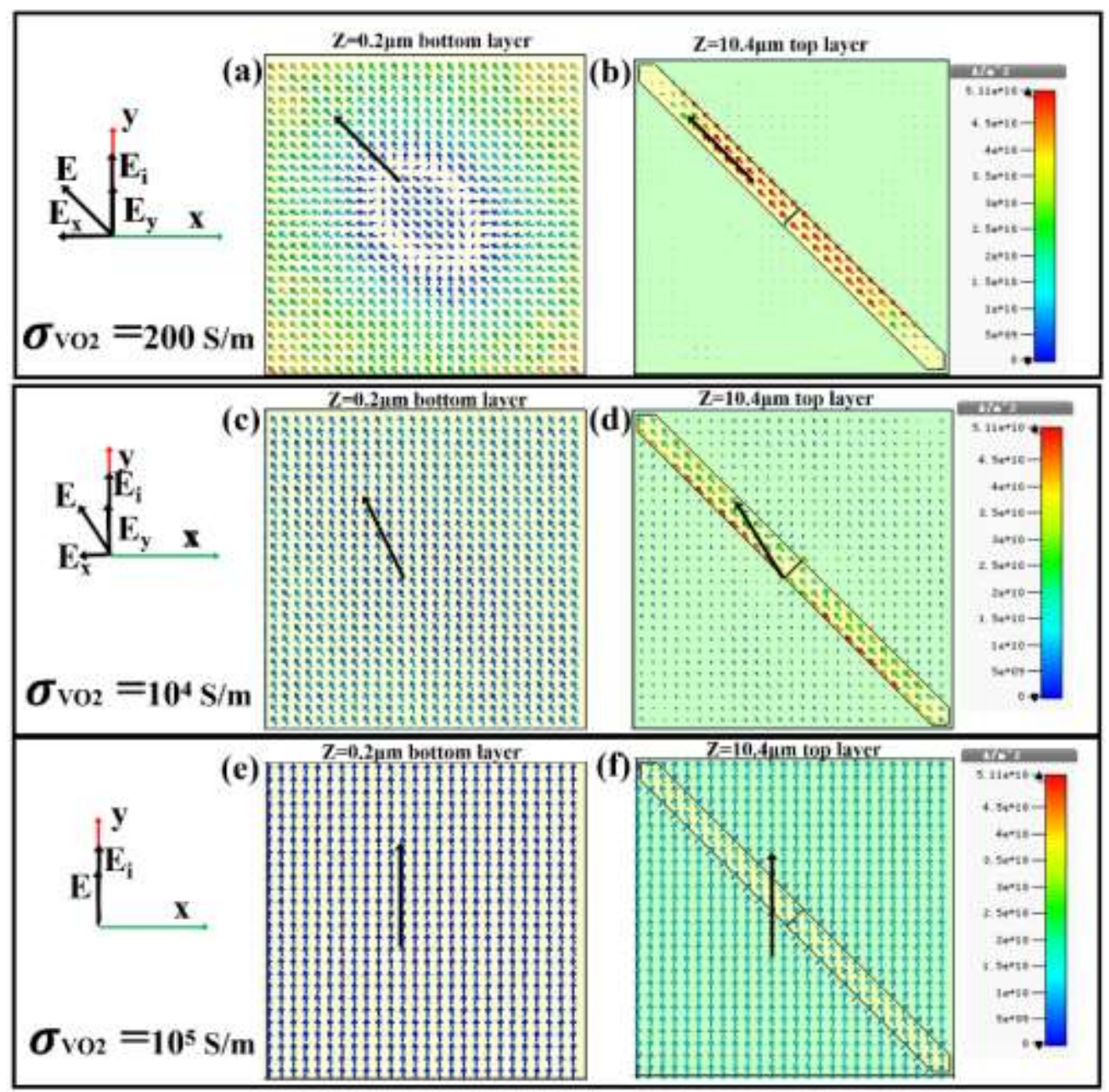

Fig. 5. Simulated current densities at $5.27 \mathrm{THz}$ at the conductivities of $200,10^{4}$ and $10^{5} \mathrm{~S} / \mathrm{m}$.

\section{Conclusions}

A simple and tunable reflective polarization converter is proposed based on metamaterial in $\mathrm{THz}$ spectrum, which composes of a two-corner-cut square patch resonator with a slit embedded into Vanadium dioxide film (VO2) and reflective ground layer. The results indicate that high PCR can be obtained for the incident linear and circular polarization wave. In addition, the dynamic thermal tunable polarization converter can also be accomplished by adjusting the conductivities of VO2. To further research, the mechanism of tunable polarization conversion is also analysed by investigating the current densities at the top and bottom layer under the condition of changeable conductivities. According to the results, the designed metamaterial could be applied in the area of temperature-controlled sensing, $\mathrm{THz}$ wireless communication, tunable polarized devices. 
Author contributions: (1) All the author have participated in conceiving the idea, designing and simulating the structure, obtaining the results. (2). All the authors gave the final approval of the version to be submitted.

Data Availability Statement: All the data generated during and/or analyzed during the current study are available from the corresponding author on reasonable request.

\section{Ethics Declarations:}

Funding: This work is supported by Major Science and Technology Project of Anhui Province in China under grant 202003a05020031 and Key research and development plan of Anhui province in China under grant 202004a05020023.

Consent to participate: All authors accepted.

Consent for publication: All authors accepted.

Competing interests: The authors declare no competing interests.

Ethics approval: All authors accepted.

\section{References}

[1] Liu H B, Zhong H, Karpowicz N, Chen Y, Zhang X C (2007) Terahertz Spectroscopy and Imaging for Defense and Security Applications. Proceedings of the IEEE 95:1514-1527

[2] O'Hara J F, Singh R, Brener I, Smirnova E, Han J, Taylor A J, Zhang W (2008) Thin-film sensing with planar terahertz metamaterials: Sensitivity and limitations. Optics Express 16:1786-1795

[3] Mousavi S A, Plum E, Shi J, Zheludev N I (2015) Coherent control of optical polarization effects in metamaterials. Sci Rep 5:8977

[4] Ma H F, Wang G Z, Kong G S, Cui TJ (2015) Independent Controls of Differently-Polarized Reflected Waves by Anisotropic Metasurfaces. Scientific Reports 5:9605

[5] Tang J, Xiao Z, Xu K, Ma X, Liu D, Wang Z (2016) Cross polarization conversion based on a new chiral spiral slot structure in THz region. Optical and Quantum Electronics 48:111

[6] Xu G, Yu F, Guo K, Shen F, Zhou H, Sun Y, Zhang X, Yin Z, Guo Z (2017) Tri-band polarization convertor based on the multi-layer metamaterial. Applied Physics A 123:654

[7] Li H, Xiao B, Li, Huang X, Yang H (2015) Multiple-band reflective polarization converter based on deformed F-shaped metamaterial. Phys Scr 90:35806

[8] Zhang J, Tian J, Xiao S, Li L (2020) Methodology for High Purity Broadband Near-Unity THz 
Linear Polarization Converter and its Switching Characteristics. IEEE Access 8:46505-46517

[9] Cheng H, Chen S,Yu P, Li J, Xie B, Li Z, Tian J (2013) Dynamically tunable broadband mid-infrared cross polarization converter based on graphene metamaterial. Applied Physics Letters 103:223102

[10] Zhang D Q, Shu F Z, Jiao Z W, Wu H W (2021) Tunable wave plates based on phase-change metasurfaces. Optics Express 29:7494

[11] Zhang K, Liu Y, Lin S, Xia F, Kong W (2020) Actively tunable bi-functional metamirror in terahertz band. Optics Letters 46:464-467

[12] Sofi M A, Saurav K, Koul S K (2021) A linear to circular polarization reconfigurable converter based on frequency selective surface. Microwave and Optical Technology Letters 63:1425-1433

[13] Yan D, Meng M, Li J, Li J, Li X (2020) Vanadium dioxide-assisted broadband absorption and linear-to-circular polarization conversion based on a single metasurface design for the terahertz wave. Optics Express 28:29843-29854

[14] Wang D, Zhang L, Gu Y, Mehmood M Q, Gong Y, Srivastava A, Jian L, Venkatesan T, Qiu C W, Hong M (2015) Switchable Ultrathin Quarter-wave Plate in Terahertz Using Active Phase-change Metasurface. Sci Rep 5:15020

[15] Liu M, Hwang H Y, Tao H, Strikwerda A C, Fan K, Keiser G R, Sternbach A J, West K G, kittiwatanakul S, Lu J, Wolf S A, Omenetto F G, Zhang X, Nelson K A, Averitt R D (2012) Terahertz-field-induced insulator-to-metal transition in vanadium dioxide metamaterial. Nature 487:7407

[16] Zhao J X, Song J L, Zhou Y, Liu Y C, Zhou J H (2020) Switching between the Functions of Half-Wave Plate and Quarter-Wave Plate Simply by Using a Vanadium Dioxide Film in a Terahertz Metamaterial. Chinese Physics Letters 37:35-39

[17] Song Z, Zhang J (2020) Achieving broadband absorption and polarization conversion in the same frequency band. Optics Express 28:2487-12497

[18] Li J, Zheng C, Li J, Zhao H (2021) Polarization-dependent and tunable absorption of terahertz wave based on anisotropic metasurface. Optics Letters 29:3284-3295

[19] Liu M, Plum E, Li H, Li S, Xu Q, Zhang X, Zhang C, Zou C, Jin B, Han J, Zhang W (2021) Temperature-Controlled Optical Activity and Negative Refractive Index. Advanced Functional Materials. Advanced Functional Materials 31:2010249 
[20] Ren Y, Zhou T, Jiang C, Tang B (2021) Thermally switching between perfect absorber and asymmetric transmission in vanadium dioxide-assisted metamaterials. Optics Express 29:7666-7679 [21] Zhang J, Wang G, Zhang B, He T, He Y, Shen J (2016) Photo-excited broadband tunable terahertz metamaterial absorber. Optical Materials Optical Materials 54:32-36

[22] Xiao Z, Zou H, Zheng X, Ling X, Wang L (2017) A tunable reflective polarization converter based on hybrid metamaterial. Optical and Quantum Electronics 49:401-401

[23] Lv T T, Li Y X, Ma H F, Zhu Z, Li Z P, Guan C Y, Shi J H, Zhang H, Cui T J (2016) Hybrid metamaterial switching for manipulating chirality based on VO2 phase transition. Sci Rep 6:23186 [24] Wang B X (2017) Quad-band terahertz metamaterial absorber based on the combining of the dipole and quadrupole resonances of two SRRs. IEEE Journal of Selected Topics in Quantum Electronics 23:4700107

[25] Hentschel M, Weiss T, Bagheri S, Giessen H (2013) coupling of solid and inverse plasmonic structures. Nano Lett 13:4428-4433

[26] Liu N, Guo H, Fu L, Kaiser S, Schweizer H, Giessen H (2008) Three-dimensional photonic metamaterials at optical frequencies. Nature materials 7:31-37

[27] Liu N, Giessen H (2010) Coupling Effects in Optical Metamaterials. Angew Chem Int Ed Engl 49:9838-9852

[28] Zhang Z, Wang J, Fu X, Jia Y, Chen H, Feng M, Zhu R, Qu S (2021) Single-layer metasurface for ultra-wideband polarization conversion: bandwidth extension via Fano resonance. Sci Rep 11:585 [29] Zhao J C, Cheng Y Z (2017) Ultra-broadband and high-efficiency reflective linear polarization convertor based on planar anisotropic metamaterial in microwave region. Optik 136:52-57 